\title{
Regeneration of Furcation Defects Treated with Porous Hydroxyapatite and Platelet-Rich Plasma: A Histological Study
}

\author{
Avaneendra Talwar ${ }^{1} \quad$ Reshma Amin², \\ ${ }^{1}$ Department of Periodontics, A.B. Shetty Memorial Institute \\ of Dental Science, Nitte (Deemed to be University) University, \\ Deralakatte, Karnataka, India \\ 2Department of Oral Pathology, A.B. Shetty Memorial Institute \\ of Dental Science, Nitte (Deemed to be University) University, \\ Deralakatte, Karnataka, India
}

J Health Allied Sci NU:2020;10:68-73

\begin{abstract}
Address for correspondence Reshma Amin, MDS, Department of Oral Pathology, A.B. Shetty Memorial Institute of Dental Science, Nitte (Deemed to be University), Deralakatte 575018, Karnataka, India (e-mail: reshyesh@yahoo.com).
\end{abstract}

\begin{abstract}
Keywords

- histology

- periodontal

regeneration

- platelet-rich plasma

Introduction The alveolar bone, periodontal ligament (PDL), and cementum are the integral structural components invariably subject to changes during periodontal diseases. Many documents on animal and human studies state that the polypeptide growth factors (GFs) have a role in periodontal regeneration. The platelet-rich fibrin (PRF) is in use since the last decade. It has the potential of delivering GFs into vertical osseous defects. Human platelets contain platelet-derived growth factor and transforming growth factor- $\beta$ in their $\alpha$ granules. These GFs are involved in wound healing and act as promoters of tissue regeneration. The study aimed to obtain histologic evidence, if any, of new attachment in humans, following treatment of osseous defects with hydroxyapatite (HA) and PRF mix.

Materials and Methods Five maxillary molar teeth with advanced bone loss were treated with porous hydroxyapatite (PHA) mixed with PRF. Distobuccal roots were resected and studied histologically for evidence of bone formation, if any, at 1st, 3rd, and the 5th months.

Results The PHA was completely resorbed in all the specimens. There was definitive evidence of bone formation at the 3rd month itself with mature lamellar bone with resting and reversal lines at the fifth postoperative month. There was no evidence of new cementum or PDL formation.

Conclusion PRF in conjunction with osteoconductive materials prove to accelerate bone formation in vertical osseous defects.
\end{abstract}

\section{Introduction}

The alveolar bone, periodontal ligament (PDL), and cementum are the integral structural components invariably subject to change during periodontal disease. Open-flap debridement promotes the growth of the microorganism community due to long junctional epithelium formation, leading to the less stable attachment. ${ }^{1}$

Bone replacement grafts are in use for a long time, to enhance bone formation and regeneration of periodontal tissues in vertical osseous defects. Bone formation occurs by processes such as osteoconduction, osteoinduction, or osteogenesis. ${ }^{2}$ Autogenous bone graft remains the gold standard, ${ }^{3}$ but postoperative morbidity and limited available volume of intraoral autogenous bone are the major drawbacks to its extensive use.

Hydroxyapatite (HA), a synthetic alloplastic bone graft, is chiefly utilized in the treatment of osseous defects. The porous form of $\mathrm{HA}$ forms through a hydrothermal conversion of the calcium carbonate of coral exoskeleton. The HA forms 
a scaffold to support the fibrovascular ingrowth with subsequent bone formation by osteoconduction. ${ }^{4}$

The lack of osteoinductive potential is a disadvantage for porous hydroxyapatite (PHA) usage in intrabony defects. ${ }^{5}$ Histological evidence of lamellar bone formation, the significant component within the pores, has been found in defects treated with PHA. ${ }^{4,6}$ However, other reports suggest encapsulation of graft material within the forming of new bone, without any evidence of its resorption., 5

Newer regeneration therapies have focused on the biomimetic approach, utilizing naturally occurring biological modifiers such as polypeptide growth factors (GFs) to accelerate wound healing. The GFs regulate several other cellular events in tissue regeneration involving chemotaxis, cell proliferation, differentiation, or matrix synthesis via binding to specific cell surface receptors. ${ }^{8}$

Recombinant GFs either singly or in combination have provided evidence for bone regeneration in periodontal defects in both animal and human trials. ${ }^{9-15}$ However, these are cost-prohibitive, thus not used clinically to a great extent.

Alternatively, platelet-rich plasma (PRP), procured from whole blood is a rich source of autologous GFs, and can promote periodontal regeneration. These GFs are approximately 300 times more than that of the levels present in normal plasma. Although plasma is considered to have more than 30 different GFs, platelet-derived growth factor (PDGF) and transforming growth factor- $\beta$ (TGF- $\beta$ ) present in platelets are the most important. They assist in tissue healing, mainly by stimulating proliferation and differentiation of mesenchymal cells. ${ }^{16,17}$ PRP with synthetic porous HA triggers the formation of new bone in the healing site by the release of GFs. The osteoinductive and osteoconductive functions of the HA stimulate the differentiation of osteoblastic/progenitor cells.

Histological evidence of new attachment in humans is sparse. Nevins et al were the first to demonstrate periodontal regeneration, including bone, PDL, and the cementum in human histological specimens of severe osseous defects following treatment with recombinant PDGF. ${ }^{18}$

This study aimed to obtain histologic evidence, if any, of new attachment in humans, following treatment of osseous defects with HA and PRP mix.

\section{Materials and Methods}

\section{Patient Selection}

Following an ethical committee consent, five adult patients $24-53$ years of age were enrolled in the study. Patients presenting clinically with probing pocket depth of 5-8 $\mathrm{mm}$ and a clinical attachment level greater than $5 \mathrm{~mm}$, and with radiographic evidence of at least one advanced osseous defect involving the distobuccal root up to the apical third with a vertical component of at least $5 \mathrm{~mm}$ were selected. For the teeth associated with the osseous defects, a planning of radisection of the distobuccal root by two independent periodontists ( - Fig. 1) was done. Patients who were systemically healthy and with no contraindication to periodontal therapy were included. Patients with systemic diseases and tooth with osseous defects with good regenerative potential were excluded from the study.

\section{Presurgical Therapy}

Preoperative hematological assessment including a complete blood count was done. Initial therapy consisted of full-mouth
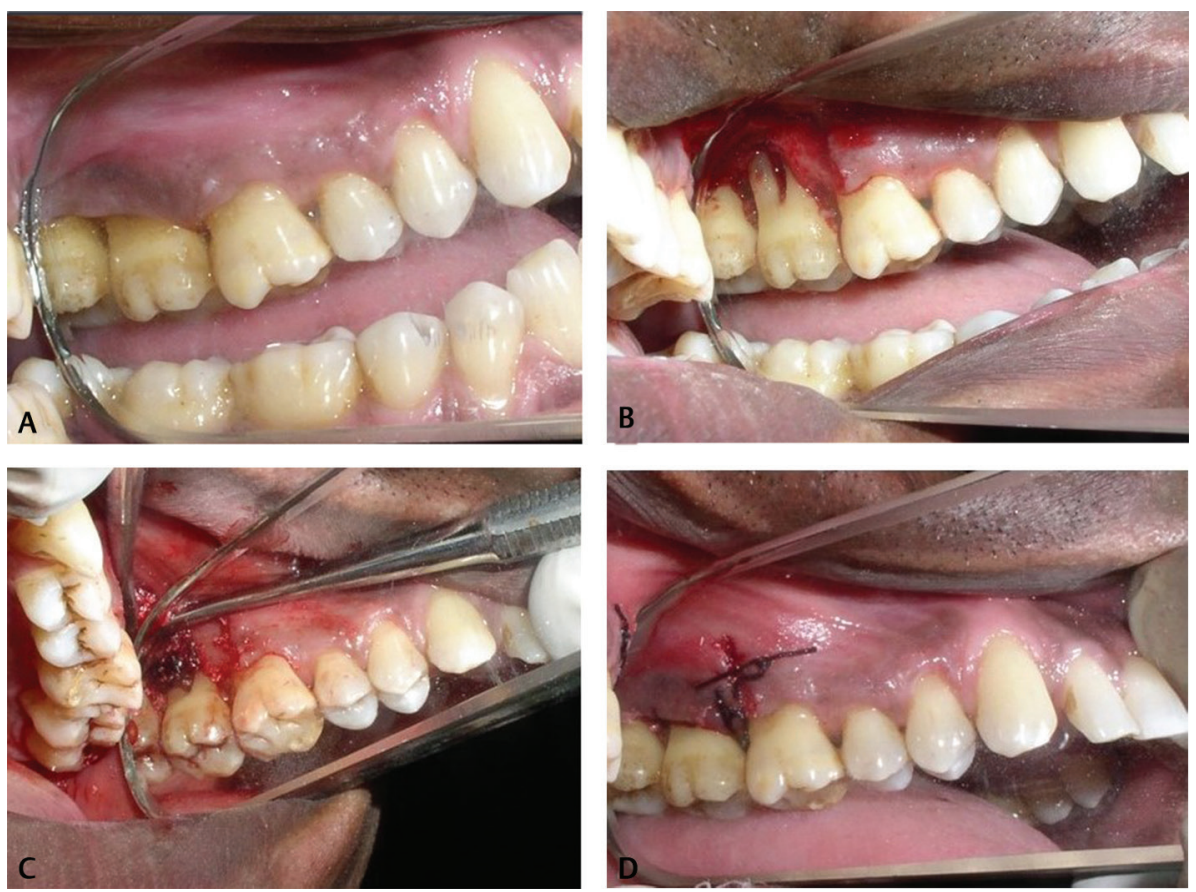

Fig. 1 (A) Preoperative clinical view. (B) Intraoperative view of defect. (C) Defect filled with platelet-rich plasma and porous hydroxyapatite. (D) Flap closure by means of interrupted sutures. 
scaling and root planning utilizing both hand and ultrasonic instruments under local anesthesia. Oral hygiene instructions were given at each visit and were reinforced throughout the study. They were repeated until the patient achieved a full-mouth plaque score of less than $25 \%$ and there was absence of clinical signs of gingival inflammation. Symptoms of trauma of occlusion, if detected, were corrected.

\section{Preparation of Platelet-Rich Plasma}

PRP was procured by the method previously described by Sonnleitner et al. ${ }^{17}$

Briefly, PRP was extracted 30 minutes prior to surgery using venipuncture. Further, $20 \mathrm{~mL}$ of blood was drawn from each patient by venipuncture of the antecubital vein in the forearm into a $20 \mathrm{~mL}$ syringe. Then, $10 \mathrm{~mL}$ of blood was collected into two glass tubes containing $10 \%$ trisodium citrate solution as an anticoagulant. The glass tubes containing blood were centrifuged at $1,200 \mathrm{rpm}$ for 20 minutes, resulting in the separation of two fractions: plasma at the top and red blood cells at the bottom. The plasma, along with the top $2 \mathrm{~mL}$ of red blood cells, was aspirated with the help of "Eppendorf pipettes." This fraction was again centrifuged at 2,000 rpm for 15 minutes to get three basic fractions: platelet-poor plasma (PPP) at the top of the preparation (supernatant), PRP in the middle, and red blood cell fraction at the bottom. The top $80 \%$ corresponding to PPP was aspirated with a pipette, leaving the residual $(0.5-2 \mathrm{~mL})$ platelet concentrate.

\section{Surgical Protocol}

The surgical procedure was performed under local anesthesia. Buccal and lingual sulcular incisions were used and a mucoperiosteal flap was elevated. Care was taken to preserve as much interproximal soft tissue as possible. Complete debridement of the defects as well as scaling and root planning were achieved with the use of an ultrasonic device and hand instruments (-Fig. 2).
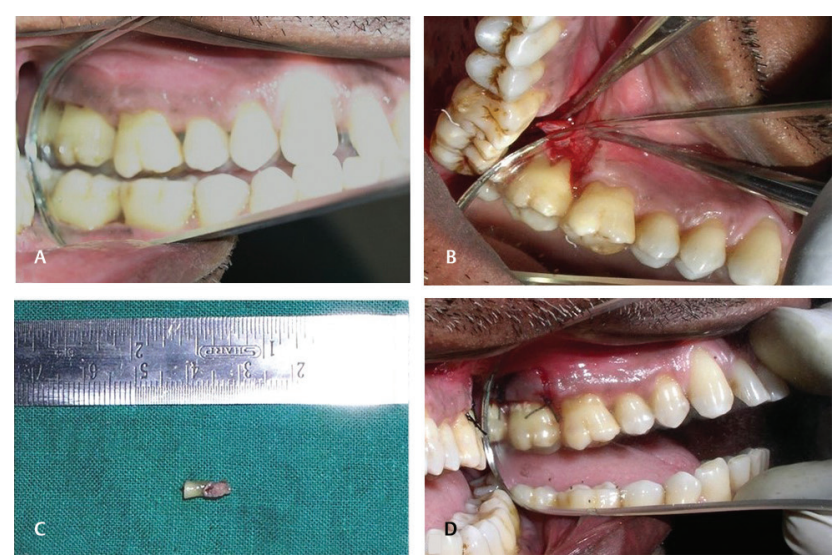

Fig. 2 (A) Five-month postoperative clinical view. (B) Reentry of the treated site. (C) Distobuccal root removed, together with some surrounding hard tissue. (D) Flap closure by means of interrupted sutures following radisection.
Intrabony defects after soft tissue debridement were measured vertically from the base and the crest of the defect to the cemento-enamel junction.

\section{Treatment of the Defect}

The bone graft was a synthetic, osteoconductive, nonceramic form of HA (Osteogen - Impladent, New York, United States) with the PRP in a proportion of 1:1. Coagulation of PRP/synthetic HA mixture was prepared with $5 \mu \mathrm{mL}$ of $10 \%$ calcium chloride, which takes up a sticky gel consistency. This mixture was then packed into the bony defects to the bony crest level (-Fig. 2C).

Flaps were sutured using interrupted sutures ( - Fig. 2D). Prescription of antibiotics (Amoxicillin $1.5 \mathrm{~g}$ every $6 \mathrm{~h}$ for $5 \mathrm{~d}$ ) and $0.12 \%$ chlorhexidine gluconate rinse (every 12 $\mathrm{h}$ for 2 weeks) along with oral analgesic (Ibuprofen $400 \mathrm{mg}$ every $8 \mathrm{~h}$ as necessary) was given.

\section{Postoperative Care}

Periodical assessment was done weekly for the 1 st month, every other week for the 2nd month, and monthly until biopsy. Suture removal was done after 2 weeks postoperatively cleansed with $0.12 \%$ chlorhexidine gluconate on a cotton swab. Patients were given guidelines for brushing at the end of the second postoperative week. They were examined weekly up to 1 month after surgery and then in the 3rd and 5th months. Postoperative precautions with care included the emphasis on oral hygiene and mechanical plaque removal, whenever mandatory. Radiographs were taken at regular intervals to check for bone formation (-Fig. 3).

\section{Radisection}

All biopsies were sent to the laboratory for analysis. Radisection of the vital tooth was performed at 1st, 3rd, and 5th months in different patients ( - Fig. $\mathbf{3 C}$ ) and the specimens fixed in $10 \%$ neutral formalin and demineralized in $20 \%$
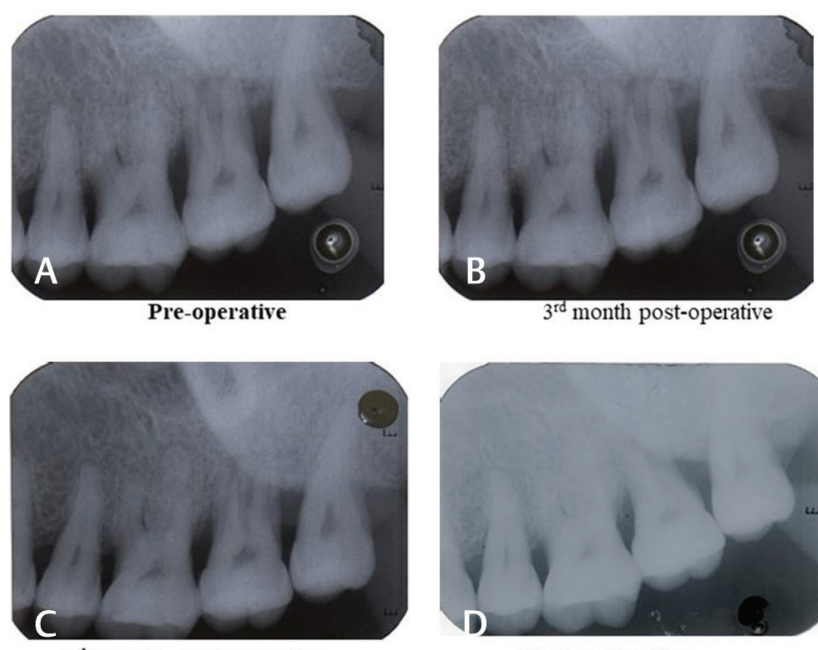

$5^{\text {th }}$ month postoperative

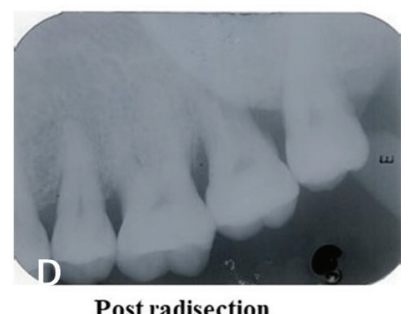

Post radisection

Fig. 3 Intraoral periapical radiographs taken at different time intervals. 
formic acid over a period of 2 months and embedded in paraffin wax. Sections $5 \mu \mathrm{m}$ thick were cut through each biopsy and were stained with hematoxylin and eosin. Stained sections were photographed under light microscope attached to a Motic (version plus 3.0). Histologic evaluations of the specimens were conducted by an oral pathologist.

The patients were asked to report after 10 days to remove the sutures and to debride the wound. After healing of the surgical sites, patients were advised to report for reviews at regular intervals to check for wound healing and vitality of the teeth.

\section{Results}

All five patients completed treatment and experienced no adverse reactions related to treatment. Clinically, wound healing appeared enhanced in PRP/PHA treated sites. Following surgery, the distobuccal roots were extracted at 1,3 , and 5 months in different patients and the specimens obtained were processed and examined under light microscope.

Histological evaluation was performed for four of the five specimens as one specimen was not evaluable due to difficulties encountered during processing.

The photomicrograph of specimens taken 1 month after grafting showed no evidence of particulate graft material. Encapsulation of graft with a fibrous tissue was also not observed. Inflammatory cell presence was sparse, with no characteristic. Mature collagen was observed in the treated area. Immature bone formation was also observed ( - Fig. 4 A).
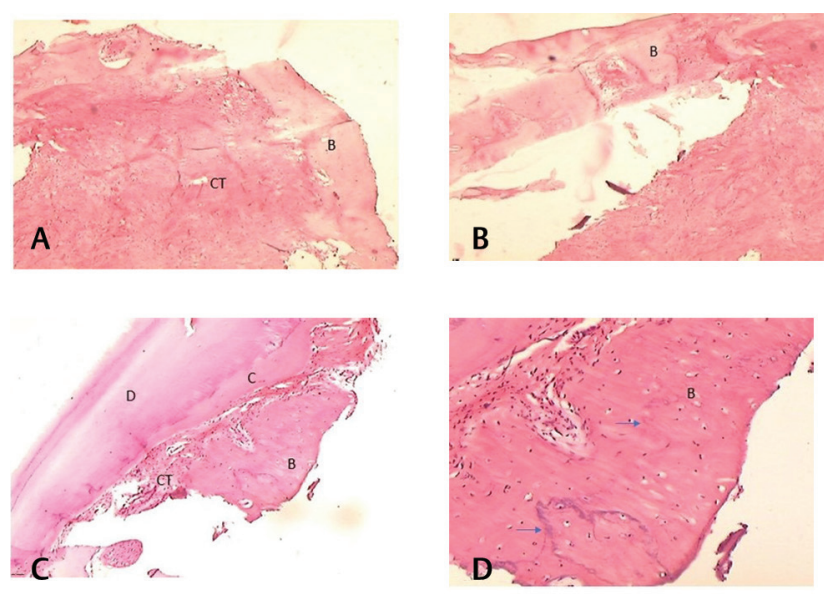

Fig. 4 (A) Photomicrograph of specimens taken 1 month after treatment shows immature bone with mature collagen. Few inflammatory cells are seen (hematoxylin-eosin stain magnification $4 \times$ ). (B) Photomicrograph of specimens taken 3 months post operation shows mature bone formation (hematoxylin-eosin stain, original magnification $4 \times$ ). (C) Photomicrograph of specimens taken at 5 months shows root bit along with presence of cellular connective tissue and mature bone (hematoxylin-eosin stain, magnification 4×). (D) Photomicrograph of higher magnification of the specimens taken at 5 months shows mature bone with osteocytes within lacunae and presence of resting lines (hematoxylin-eosin stain, magnification 10×). Note: $C T$, connective tissue; D, dentin; C, cementum; B, mature bone; Arrow, resting lines.
The photomicrograph of specimens taken at 3 months after grafting showed a small amount of mineralized bone with fibrous connective tissue ( $\boldsymbol{- \text { Fig. 4B). }}$.

The photomicrograph of specimens taken at 5 months showed mature bone with osteocytes within lacunae along with the presence of resting lines. There was also the presence of cellular connective tissue ( - Fig. 4 C and D). None of the slides showed PDL or acellular cementum.

\section{Discussion}

The limitations of traditional therapies have promoted the development of tissue engineering. This emerging field is concerned with the development of natural biological surrogates that restore, maintain, or improve tissue structure and function. Three general strategies have emerged for the engineering of tissues. The first is a conductive approach in which synthetic scaffold materials amenable to infiltration of specific cell types are implanted into a site of disease or defect. The materials provoke the conduction of desired cell types while blocking the conduction of unwanted cell types. The second approach involves the inclusion of bioactive factors (e.g., GFs) into the aforementioned synthetic scaffolds. They are chosen to spur the infiltration of the specific cell types and induce the formation of any particular type of tissue. The third approach is on the basis of seeding scaffolds with cells in vitro, followed by implantation of the cell construct. ${ }^{19}$

Basic and clinical research has focused on the application of GFs for the regeneration of tissues. This can be achieved through gene therapy. In a review, Yao and Eriksson reported that short shelf life and inefficient delivery to target cells are the major concerns associated with local administration of recombinant human GFs. The GFs were expensive and sufficient doses were required to achieve any obvious therapeutic effect. ${ }^{20}$

Another easy, cost-effective way to obtain concentrations of GFs for tissue healing and regeneration may be autologous platelet storage via PRP.

GFs such as TGF- $\beta$ and PDGF determine the rationalization of the use of PRP as a bone regenerative stimulating agent. These are carried into the regenerating site with an ideal carrier, like the patient's platelets. ${ }^{8}$

The ability of PRP to enhance the consolidation of bone graft has been well established since 1998 by the pioneering works of Marx et al..$^{21,22}$

Several studies have exemplified the role of platelet formulations in regeneration of soft/hard tissues, including formation of new bone. In these studies, different types of bone replacement grafts such as demineralized bone powder, Biobone/Bio-Oss, HA, and other forms of allografts have been used in combination with PRP gel in the treatment of osseous defects. ${ }^{23,24}$

During the bone regeneration process, the GFs present in the PRP gel carry out important functions for the initiation and maintenance of the proliferation, and differentiation of the osteoblastic precursor cells and osteoblasts themselves, leading to bone formation. ${ }^{22}$ 
In this study, the PRP was prepared using a method previously described, except that thrombin was not used to avoid any risk to the patient by mixing PRP with any component of animal or human origin. ${ }^{25,26}$

Nevins et $\mathrm{al}^{18}$ demonstrated periodontal regeneration including bone PDL and cementum after 9 months in sites treated with recombinant human PDGF. However, the dynamics of the healing process were not studied as all the specimens were obtained after 9 months. To gain further insight into the regenerative process, the specimens in this study were obtained at 1,3 , and 5 months of intervals.

In our study, the photomicrograph of a specimen taken at 1 month after grafting showed the presence of osteoid formation, with mature collagen and sparse inflammatory cells. There was no evidence of fibrous encapsulation of the graft or remnants of graft material. Previous histologic studies have showed that though the use of porous synthetic HA resulted in bone formation, there was limited osteogenesis with the graft particles being encapsulated in the new bone formed. , $, 5,7,27$

This early resorption of PHA particles in this study could be the result of increased inflammatory cell infiltrate following PRP application, as suggested by Hartwig et al. ${ }^{28}$ Stimulated platelets actively synthesize proinflammatory cytokines (e.g., CD40L, IL- $\beta$ ) and activate macrophages by the release of chemokines. The increased number of macrophages may have accelerated the resorption of the bone graft.

However, at the 3rd-month stage, this inflammatory cell exudate did not translate into excessive osteoclastic activity. The presence of collagen and bone formation at the healing site is suggestive of more osteoblastic than osteoclastic activity. Kubota et $\mathrm{al}^{29}$ in an in-vivo study have shown osteoclastic activation by PDGF-BB released in an inflammatory environment. However, this in-vivo result may not be truly reflective of the in-vitro healing events in a vertical osseous defect.

The subsequent slides have shown the maturation of the newly formed bone, and, at the 5th month, definite evidence of resting lines in the newly formed bone with osteocytes in the matrix suggests bone formation by osteoblastic activity.

We may summarize the results as follows:

- Early and complete resorption of the PHA alloplastic graft in all the specimens.

- Definitive evidence of bone formation started at about the 3rd month with mature bone forming within 5 months.

Current opinion about the use of PRP in regenerative procedures is not complimentary to the PRP gel. We propose that the positive results obtained in this study could be the result of the following:

- Periodontal defects being much smaller in size than oral surgical defects consume much less PRP gel. Thus PDGF-BB, which is the major component of PRP with osteoclastic activity, may be in smaller concentrations. PDGF-BB has also been shown to have a very short halflife $^{30}$ and hence, the osteoclastic activation may not be of much clinical significance.
- When mixed with a slow resorbing alloplast like PHA, the action of PRP may be prolonged for an interval sufficient enough to release PDGF and TGF- $\beta$ and hence induce osteoblastic activation.

- The early evidence of bone formation at the 3rd month itself is clear evidence of bone formation as a result of PRP and not just the PHA alloplast.

In this study, there was no evidence of PDL formation as shown by Nevins et al $^{18}$ probably because:

- The PHA mixed with PRP may have precluded the possibility of the fibrin glue adhering to the root and hence may not have impeded apical migration of junctional epithelium.

- The experimental sites were not ideal conditions for regeneration due to their extensive bone loss and gingival recession.

It may thus be a fair assumption to state that in defects with better prognosis, the regenerative potential would only increase. A more precise understanding of the dynamics of GF release from PRP used in smaller periodontal defects versus more extensive surgical wounds would help to clarify their exact role in osteoblast/osteoclast activation. The evidence of this study would seem to indicate that some of the criticism about PRP in periodontal defect treatment is unwarranted.

In conclusion, platelet-rich fibrin in conjunction with osteoconductive materials prove to accelerate bone formation in vertical osseous defects.

\section{Conflict of Interest}

None declared.

\section{References}

1 Garrett S. Periodontal regeneration around natural teeth. Ann Periodontol 1996;1(1):621-666

2 Mellonig JT. Autogenous and allogeneic bone grafts in periodontal therapy. Crit Rev Oral Biol Med 1992;3(4):333-352

3 Bowers GM, Chadroff B, Carnevale R, et al. Histologic evaluation of new attachment apparatus formation in humans. Part II. J Periodontol 1989;60(12):675-682

4 Kenney EB, Lekovic V, Sa Ferreira JC, Han T, Dimitrijevic B, Carranza FA Jr. Bone formation within porous hydroxylapatite implants in human periodontal defects. J Periodontol 1986;57(2):76-83

5 Stahl SS, Froum SJ. Histologic and clinical responses to porous hydroxylapatite implants in human periodontal defects. Three to twelve months postimplantation. J Periodontol 1987;58(10):689-695

6 West TL, Brustein DD. Freeze-dried bone and coralline implants compared in the dog. J Periodontol 1985;56(6):348-351

7 Martin RB, Chapman MW, Holmes RE, et al. Effects of bone ingrowth on the strength and non-invasive assessment of a coralline hydroxyapatite material. Biomaterials 1989;10(7):481-488

8 McCauley LK, Somerman MJ, Biological modifiers in periodontal regeneration. Advances in Periodontics, Part-1. In: Vandersall DC, ed. Dental Clinics of North America. April 1998; 42(2): 361-387

9 Gamal AY, Mailhot JM. The effect of local delivery of PDGF-BB on attachment of human periodontal ligament fibroblasts to periodontitis-affected root surfaces-in vitro. J Clin Periodontol 2000;27(5):347-353

10 Giannobile WV, Hernandez RA, Finkelman RD, et al. Comparative effects of platelet-derived growth factor-BB 
and insulin-like growth factor-I, individually and in combination, on periodontal regeneration in Macaca fascicularis. J Periodontal Res 1996;31(5):301-312

11 Howell TH, Fiorellini JP, Paquette DW, Offenbacher S, Giannobile WV, Lynch SE. A phase I/II clinical trial to evaluate a combination of recombinant human platelet-derived growth factor-BB and recombinant human insulin-like growth factor-I in patients with periodontal disease. J Periodontol 1997;68(12):1186-1193

12 Lynch SE, de Castilla GR, Williams RC, et al. The effects of short-term application of a combination of platelet-derived and insulin-like growth factors on periodontal wound healing. J Periodontol 1991;62(7):458-467

13 Lynch SE, Williams RC, Polson AM, et al. A combination of platelet-derived and insulin-like growth factors enhances periodontal regeneration. J Clin Periodontol 1989;16(8):545-548

14 Takayama S, Murakami S, Miki Y, et al. Effects of basic fibroblast growth factor on human periodontal ligament cells. J Periodontal Res 1997;32(8):667-675

15 Rutherford RB, Niekrash CE, Kennedy JE, Charette MF. Plateletderived and insulin-like growth factors stimulate regeneration of periodontal attachment in monkeys. J Periodontal Res 1992;27(4 Pt 1 ) :285-290

16 Lynch SE, Introduction. In: Lynch SE, Genco RJ, Marx RE, eds. Tissue Engineering Applications in Maxillofacial Surgery and Periodontics. Chicago: Quintessence Publishing Co, Inc; 1999: xi-xviii

17 Sonnleitner D, Huemer P, Sullivan DY. A simplified technique for producing platelet-rich plasma and platelet concentrate for intraoral bone grafting techniques: a technical note. Int J Oral Maxillofac Implants 2000;15(6):879-882

18 Nevins M, Camelo M, Nevins ML, Schenk RK, Lynch SE. Periodontal regeneration in humans using recombinant human platelet-derived growth factor-BB (rhPDGF-BB) and allogenic bone. J Periodontol 2003;74(9):1282-1292

19 Murphy WL, Mooney DJ. Controlled delivery of inductive proteins, plasmid DNA and cells from tissue engineering matrices. J Periodontal Res 1999;34(7):413-419
20 Yao F, Eriksson E. Gene therapy in wound repair and regeneration. Wound Repair Regen 2000;8(6):443-451

21 Marx RE, Carlson ER, Eichstaedt RM, Schimmele SR, Strauss JE, Georgeff KR. Platelet rich plasma: growth factor enhancement for grafts. Oral Surg Oral Med Oral Pathol Radiol Endod 1998;85(6):638-646

22 Marx RE. Platelet-rich plasma (PRP): what is PRP and what is not PRP? Implant Dent 2001;10(4):225-228

23 Siebrecht MA, De Rooij PP, Arm DM, Olsson ML, Aspenberg P. Platelet concentrate increases bone ingrowth into porous hydroxyapatite. Orthopedics 2002;25(2):169-172

24 Lekovic V, Camargo PM, Weinlaender M, Vasilic N, Kenney EB. Comparison of platelet-rich plasma, bovine porous bone mineral, and guided tissue regeneration versus platelet-rich plasma and bovine porous bone mineral in the treatment of intrabony defects: a reentry study. J Periodontol 2002;73(2):198-205

25 Anitua E. Plasma rich in growth factors: preliminary results of use in the preparation of future sites for implants. Int J Oral Maxillofac Implants 1999;14(4):529-535

26 Landesberg R, Moses M, Karpapkin M. Risks of using platelet rich plasma gel. To the editor. J Oral Maxillofac Surg 1998;56:116-117

27 Carranza FA Jr, Kenney EB, Lekovic V, Talamante E, Valencia J, Dimitrijevic B. Histologic study of healing of human periodontal defects after placement of porous hydroxylapatite implants. J Periodontol 1987;58(10):682-688

28 Hartwig D, Härtel C, Hennig H, Müller-Steinhardt $\mathrm{M}$, Schlenke P, Klüter H. Evidence for de novo synthesis of cytokines and chemokines in platelet concentrates. Vox Sang 2002;82(4):182-190

29 Kubota K, Sakikawa C, Katsumata M, Nakamura T, Wakabayashi K. Platelet-derived growth factor BB secreted from osteoclasts acts as an osteoblastogenesis inhibitory factor. J Bone Miner Res 2002;17(2):257-265

30 Bowen-Pope DF, Malpass TW, Foster DM, Ross R. Plateletderived growth factor in vivo: levels, activity, and rate of clearance. Blood 1984;64(2):458-469 